\title{
Staple line leak with peritonitis after laparoscopic sleeve gastrectomy - a solution in one to six steps
}

\author{
Petr Špička \\ Department of Surgery, Prostějov Hospital, Prostějov, Czech Republic
}

Videosurgery Miniinv 2017; 12 (2): 154-159

DOI: https://doi.org/10.5114/wiitm.2017.68297

\begin{abstract}
Introduction: Laparoscopic sleeve gastrectomy (LSG) is one of the most efficient bariatric interventions in morbidly obese patients. The most severe risk of this procedure seems to be the staple line leak, and the management of this complication can be very arduous.

Aim: To share our experience in managing the staple line leak after LSG and to help to find the best procedure that should be preferred.

Material and methods: In the 2010-2015 period we performed 223 LSG, with about 5 demonstrating severe complications - two patients with severe bleeding requiring revision surgery, and three patients with resection surface leak. Results: We always primarily treated the staple line leak with a laparoscopic revision. Once the fistula did not spontaneously close after this treatment. A series of other methods were then indicated for this patient and only the sixth one resulted in the desirable therapeutic success. At first, our team opted for laparoscopic revision with drainage. The next procedure involved applying Ovesco and Boston clips. As a third method we performed abscess drainage through a nasobiliary tube inserted via gastroscopy. Due to failure we performed the second laparoscopic revision with staple line resuture, the next intervention was an open revision with fistula excision and suture, and finally we opted for the application of a self-expanding metallic stent, which proved to be definitely curative.

Conclusions: Without any guidelines it is very difficult to determine the right procedure addressing the staple line leak after LSG. It depends mainly on the clinician's experience and is lengthy and often untraditional.
\end{abstract}

Key words: laparoscopic sleeve gastrectomy, staple line, leak.

\section{Introduction}

Laparoscopic sleeve gastrectomy (LSG) is considered as one of the most efficient bariatric interventions in morbid obesity. Laparoscopic sleeve gastrectomy involves a longitudinal resection of the stomach on the greater curvature, commonly using 3 to 5 staples. As a safe procedure, it is well tolerated by patients, involves short hospitalization, and its effect is usually reported as reliable [1]. Nevertheless, as with every surgery there are some risks, and perhaps the most severe is a leak along the staple line, reported in $1.1 \%$ to $6.5 \%$ of cases. Depending on the extent and general and local factors, the leak results in peritonitis requiring immediate revisional intervention. Many methods pertaining to successful leak management have been described globally; however, there are no set guidelines to date as the best option of leak management. Given the complexity of the diagnosis itself and the concerned bariatric patients, it is even more unlikely. As a result, every procedure is "tailored" to a specific patient and it always depends on the experience of the respective institution to

\section{Address for correspondence}

Petr Špička MD, Department of Surgery, Prostějov Hospital, 291/1 Mathonova St, 79604 Prostějov, Czech Republic,

phone: +42 0774602818, e-mail: pspicka@email.cz 
select the method and to decide whether the procedure is curative or otherwise.

\section{Aim}

The objective of this paper is to share the author's experience in managing LSG staple line leak with the general surgical community and thus contribute to the global discussion on this issue and to the quest to find the best option as the preferred procedure. Concurrently, the paper aims to highlight the complications that may arise if the leak is not secured at the first attempt, as well as the longitudinal perspective of managing such complications.

\section{Material and methods}

Since 2010, our surgery has performed 40 LSG procedures on average per year. Of the 223 LSG procedures conducted in the period 2010-2015, severe complications developed in 5 cases -2 patients with severe bleeding requiring revisional surgery and 3 patients with resection surface leaks; no severe complications were reported in other patients. Leaks were always primarily treated with revisional laparoscopy and drainage and healing with a nasogastric tube. In only 1 patient the fistula did not close spontaneously after this procedure. A series of methods were then indicated for this patient; therapeutic success was achieved at the sixth attempt. The total treatment duration for this patient was 16 months. No patient in our population developed diffuse peritonitis (Table I).

\section{Results}

Two patients with post-LSG leak (body mass index (BMI) $42 \mathrm{~kg} / \mathrm{m}^{2}$ and BMI $39 \mathrm{~kg} / \mathrm{m}^{2}$ respectively) underwent primary revisional laparoscopy with drainage of the subphrenic abscess along with parenteral and nasogastric intubation; complete cure was achieved. In the third patient (BMI $\left.41 \mathrm{~kg} / \mathrm{m}^{2}\right)$, a 27 -year-old woman without any comorbidity, this procedure failed to manage the leak. Following surgery, conducted on 14 May 2014, the initial results were positive: normal laboratory test results, normal contrast medium $X$-ray of stomach, no signs of leak. The patient was discharged on day 4 in generally good condition. She returned to the clinic on day 9 following the operation for removal of stitches with no subjective problems. However, on day 14 she returned again complaining of stomach and left shoulder pain and subfebrile temperature. Laboratory tests showed elevated C-reactive protein (CRP) $(185 \mathrm{mg} / \mathrm{l})$ and leucocyte count of $14 \times$ $10^{-9} / \mathrm{l}$. Stomach X-ray with contrast medium was performed to exclude leak. Abdominal ultrasound was negative, as well as the abdominal clinical findings. Due to persistent subfebrile temperatures, abdominal computed tomography (CT) was ordered, revealing an abscess of size $55 \times 50 \times 42 \mathrm{~mm}$ under the left liver lobe (Photo 1).

The patient was immediately prepared for revisional laparoscopic surgery. Adhesions were discovered at the upper pole of the resected stomach. No fistula was detected after detailed search. The abscess was evacuated and drained. Follow-up X-ray was conducted a week after the revisional surgery, revealing persisting leak with the drain tube intact and well placed. The patient remained on total parenteral nutrition with nasogastric intubation. Two weeks following the revisional laparoscopy, CT scan demonstrated mild regression of the abscess cavity but with persisting leak. Gastroscopy was instituted on the same day, revealing a fistula orifice. An enteral feeding tube was introduced into the jejunum. Following consultation, endoscopic treatment of the fistula orifice with an Ovesco clip was suggested (Photo 2). This procedure was performed on 30 June, i.e. approximately 45 days after the primary surgery. Since the fistula was $7 \mathrm{~mm}$ in size, the procedure also entailed argon plasma coagulation and two additional Boston clips (Photo 3).

A partial effect was obtained with the clips. Over the following days, the leak subsided and the patient remained on permanent enteral nutrition. The

Table I. Number of procedures and severe complications

\begin{tabular}{|lcccccc|}
\hline Variable & 2010 & 2011 & 2012 & 2013 & 2014 & 2015 \\
\hline Bleeding & 1 & 0 & 0 & 1 & 0 & 0 \\
\hline Diffuse peritonitis & 0 & 0 & 0 & 0 & 0 & 0 \\
\hline Local peritonitis & 1 & 0 & 1 & 0 & 1 & 0 \\
\hline
\end{tabular}




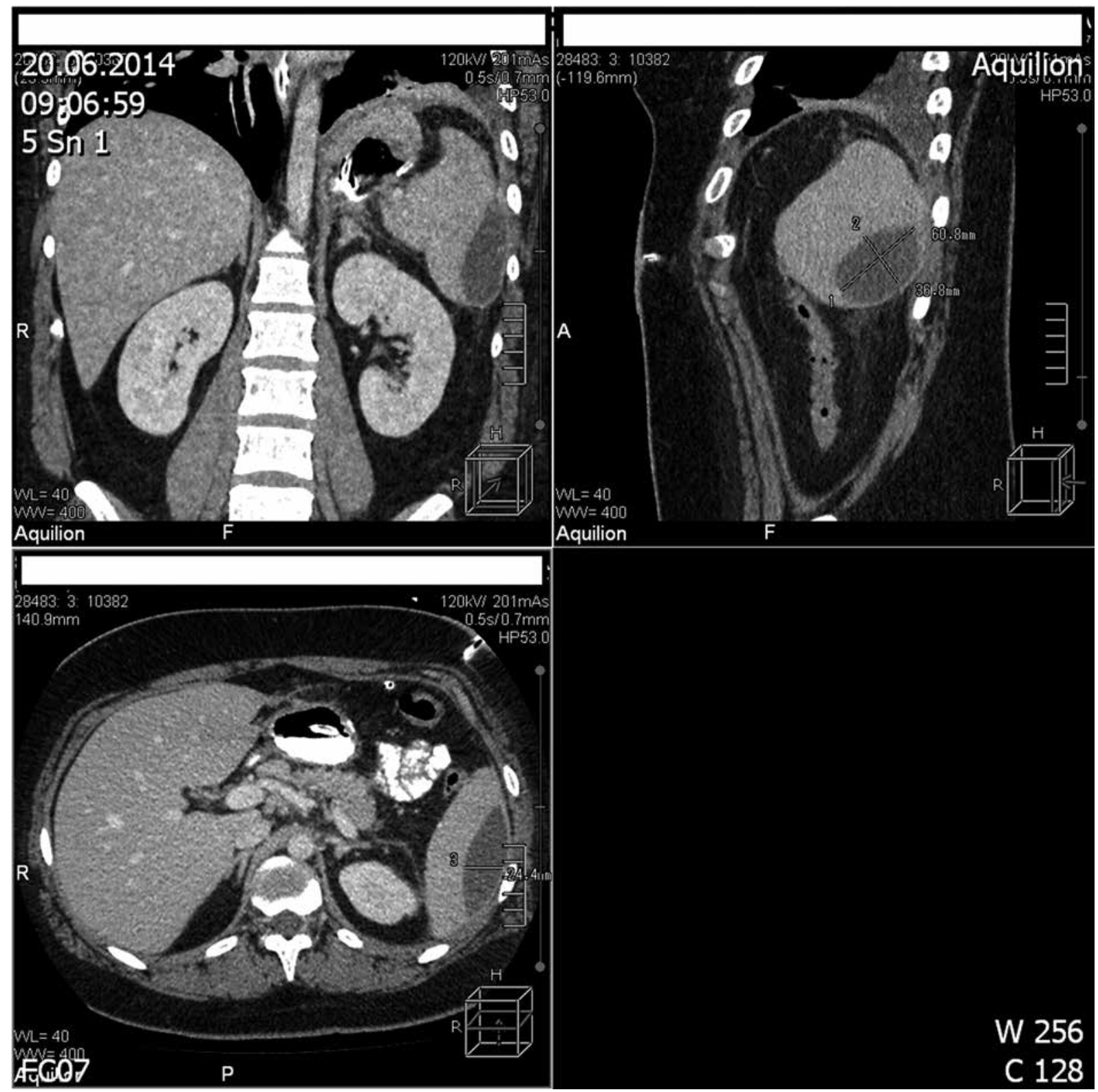

Photo 1. Leak on computed tomography scan

drain tube in the abscess, inserted at revisional laparoscopy, was gradually retracted with daily flushing and finally removed. Laboratory results were within normal limits; clinically, the patient demonstrated no significant problems. The patient was discharged with the inserted enteral nutrition tube for outpatient therapy. Follow-up CT scan performed one month after the clip application showed regression of the abscess cavity with no evidence of leak. The enteral tube was removed and the patient was returned to a normal diet. In the following 5 months, the patient experienced no problems. However, in January 2015 CT scan revealed a fistula and leak relapse. The Ovesco clip was no longer visible on CT scan; possibly, it had separated and passed per vias naturales. As another attempt to heal the fistula, gastroscopic drainage of the abscess through a nasobiliary drain tube was indicated. The tube was regularly flushed. However, following gastroscopies showed this effort to be insufficient and subsequent endoscopic treatment was not possible due to the fistula size and its unfavourable location in the car- 


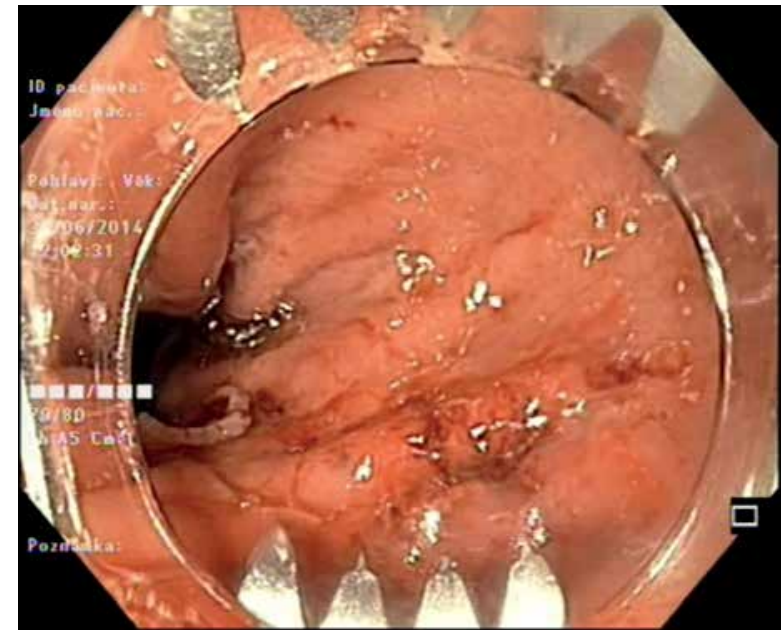

Photo 2. Ovesco clip

dia. As a result, the patient was indicated for revision surgery 1 year after the primary procedure and another revisional laparoscopy was performed, this time oversewing with an omental patch. Another CT scan one month after the operation diagnosed a stomach suture failure and a new gastrobronchial fistula (Photo 4).

Following detailed consultation, the patient was referred to a higher medical facility for revisional laparotomy comprising fistula excision, suture and gastric stump patch. On day 10, CT scan once again revealed suture failure. After expert consultation with the inhouse gastroenterologists, an esophageal self-expanding metal stent was implanted. Combined with enteral nutrition through jejunostomy conducted during laparotomy, the fistula healed inclusive of the pulmonary part. Full peroral intake was initiated and the jejunostomy was removed. Currently, the patient has been symptom free for about 11 months. She recently underwent abdominoplasty to correct the resultant abdominal contour deformity after massive weight loss. Her current BMI is $27 \mathrm{~kg} / \mathrm{m}^{2}$.

\section{Discussion}

Laparoscopic sleeve gastrectomy is at risk of resection line leaks in $1.1 \%$ to $6.5 \%$ of cases according to world literature [2-4]. In terms of pathogenesis, the leak is caused by a combination of: a) local factors - partial stomach wall ischemia, amount of stomach tissue caught in the staple, size of the staples, and staple failure, and b) general factors - metabolic disorders, abdominal obesity, etc. Another important and frequent cause of the leak is the limited

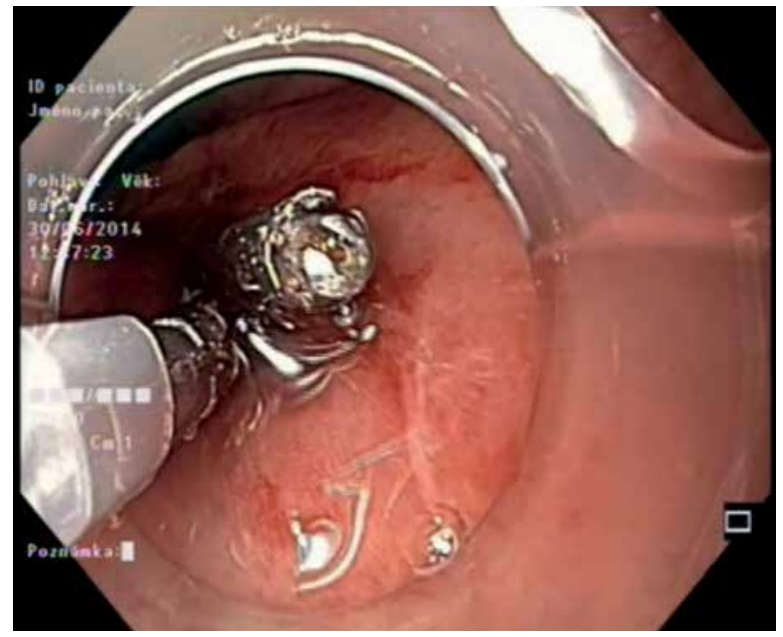

Photo 3. Boston clip

experience of the surgeon [5]. Although LSG enjoys a high success rate and life-threatening staple line leaks (SLL) are fortunately rare, their management and treatment is often complicated and the standard diagnostic and therapeutic procedures often fail in SLL patients. Staple line leaks management currently entails either a conservative approach, i.e. drainage alone with parenteral or enteral nutrition until the fistula heals, or a surgical approach that usually comprises revisional laparoscopy for drainage alone or laparoscopic re-suturing plus drainage, "re-sleeve resection" or stomach suture and conversion to Roux-en-Y gastric bypass (RYGB) [6-8]. At the extreme end of the scale is total gastrectomy

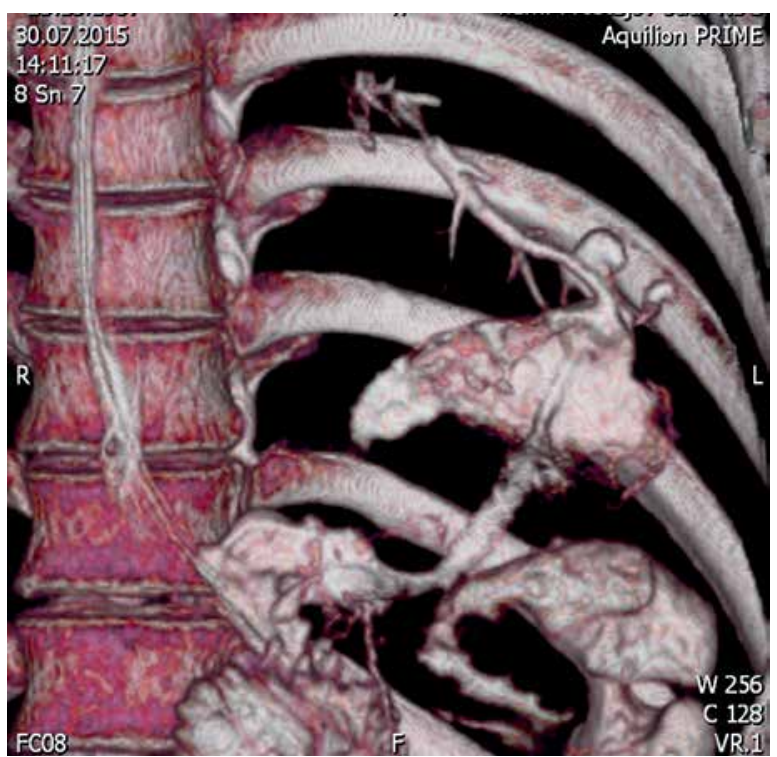

Photo 4. Gastrobronchial fistula 
[9]. Esophageal self-expanding metal stent implantation is another well-established method either on its own or complementing other procedures, with or without percutaneous glue [10-12]. Gastroscopic implantation of the Over-The-Scope Ovesco clip at the fistula orifice is somewhere on the borderline between the conservative and surgical approaches [13]. Another option is the rendezvous technique of inserting a pigtail catheter or a nasobiliary tube into the abscess cavity through the fistula, draining the abdominal cavity as well as the stomach [14]. The e-VAC system with gastroscopy offers another, rather rare, solution [15].

Given the above options, there are no set guidelines to date addressing SLL after LSG, and it is up to the experience of each surgical unit and the available data from evidence-based medicine to determine the best course of action in each case. Our patient experienced staple failure at the gastroesophageal (GE) end of the staple line of the primary procedure, which was immediately treated with replacement staples and re-stapling the resection line with perioperative evidence of suture tightness using methylene blue administered with a nasogastric tube. Upon discovering the leak, we opted for a mini-invasive procedure - revisional laparoscopy with drainage and enteral nutrition. Initially, it seemed sufficient as fistula re-suturing was not possible due to local peritonitis and location under the cardia. Our choice of this initial method was based on the available literature pertaining to resolving postoperative complications following LSG - local peritonitis, abscess. The stomach was evacuated via the nasogastric tube and the patient was initially put on full parenteral nutrition followed by enteral nutrition. However, this procedure failed and, following consultation with gastroenterologists, we opted for Over-The-Scope Ovesco clip method. Endoscopic closure combined with clips proved to be successful. With enteral nutrition in progress, the fistula healed. Some 5 months after starting peroral food intake, the fistula reappeared pursuant to local and general factors. Based on the clinical and professional experience of our gastroenterologists, we opted for the endoscopic-gastroscopic approach. A pigtail catheter was inserted into the abscess cavity to flush and drain the cavity, allowing for the fistula to close spontaneously or with the assistance of gastroscopy. However, the fistula orifice failed to be reduced and applying clips via gastroscopy was not possible. Next, we opted for laparoscopic gastroscopy-navigated fistula suture with an omental patch that was done approximately 1 year after the primary sleeve gastrectomy. In about a month, CT scan showed fistula relapse with a concomitant gastrobronchial fistula. The patient was referred to a higher medical facility where she underwent surgery. Even here, open revision with fistula extirpation and suturing failed. Following general professional consensus, a self-expanding metal stent was implanted that finally resulted in healing both the original fistula and the gastrobronchial fistula. Self-expanding metal stent implantation was, in fact, considered earlier, but the endoscopist declined to conduct the procedure owing to bad personal experience - esophageal stenosis and stent dislocation. In our patient, these complications did not occur.

\section{Conclusions}

Treating SLL after LSG is an arduous task and has to be "tailored" to each patient. No explicit and binding guidelines can, therefore, be established or published as a matter of rule. A bariatric patient, in many respects, is a complicated and usually polymorbid case. This means that the instituted treatment must be aggressive while taking into account the patient's limited reserves and tolerance. The treatment of SLL following LSG offers a wide spectrum of options, from conservative to radically surgi$\mathrm{cal}$, with the literature describing successes and failures of each method. Our first choice of revisional laparoscopy with drainage and full parental nutrition and nasogastric stomach emptying, usually referred to as highly curative, failed for our patient. The same fate was met with Over-The-Scope Ovesco clips, pigtail drainage and open resection and fistula suturing. In the end, success in our patient was achieved at the sixth attempt with self-expanding metal stent implantation.

This paper clearly demonstrates that in the absence of collective guidelines, given that such in itself is an uphill task, it is very difficult to determine the best approach when addressing the serious complications of LSG. At the moment, it relies on clinical experience, and if the first attempt to resolve the leak proves unsuccessful, the following solution(s) is/are usually very arduous, lengthy and often quite unorthodox. 


\section{Conflict of interest}

\section{The author declares no conflict of interest.}

\section{References}

1. Gajbhiye R, Tirpude B, Bhanarkar $H$, et al. A study on role of laparoscopic sleeve gastrectomy in the management of morbid obesity. Indian J Surg 2016; 78: 177-81.

2. Iossa A, Abdelgawad M, Watkins BM, et al. Leaks after laparoscopic sleeve gastrectomy: overview of pathogenesis and risk factors. Langenbecks Arch Surg 2016; 401: 757-66.

3. Ghosh SK, Roy S, Chekan E, et al. A narrative of intraoperative staple line leaks and bleeds during bariatric surgery. Obes Surg 2016; 26: 1601-6.

4. Stroh C, Köckerling F, Volker L, et al. Obesity Surgery Working Group, Competence Network Obesity. Results of more than 11,800 sleeve gastrectomies: data analysis of the German Bariatric Surgery Registry. Ann Surg 2016; 263: 949-55.

5. Noel P, Nedelcu M, Gagner M. Impact of the surgical experience on leak rate after laparoscopic sleeve gastrectomy. Obes Surg 2016; 26: 1782-7.

6. Praveenraj P, Gomes RM, Kumar S, et al. Management of gastric leaks after laparoscopic sleeve gastrectomy for morbid obesity: a tertiary care experience and design of a management algorithm. J Minim Access Surg 2016; 12: 342-9.

7. Mahmoud M, Maasher A, Al Hadad M, et al. Laparoscopic Roux En Y esophago-jejunostomy for chronic leak/fistula after laparoscopic sleeve gastrectomy. Obes Surg 2016; 26: 679-82.

8. Chouillard E, Younan A, Alkandari M, et al. Roux-en-Y fistulo-jejunostomy as a salvage procedure in patients with post-sleeve gastrectomy fistula: mid-term results. Surg Endosc 2016; 10: 4200-4.

9. Zanotti D, Elkalaawy M, Mohammadi B, et al. Gastro-cutaneous fistula 4 years after a fully resolved staple line leak in sleeve gastrectomy. J Surg Case Rep 2015; 2015: rjv152.

10. Southwell T, Lim TH, Ogra R. Endoscopic therapy for treatment of staple line leaks post-laparoscopic sleeve gastrectomy (LSG): experience from a Large Bariatric Surgery Centre in New Zealand. Obes Surg 2016; 26: 1155-62.

11. Galloro G, Ruggiero S, Russo T, et al. Staple-line leak after sleve gastrectomy in obese patients: a hot topic in bariatric surgery. World J Gastrointest Endosc 2015; 7: 843-6.

12. Vilallonga R, Himpens J, Bosch B, van et al. J. Role of percutaneous glue treatment after persisting leak after laparoscopic sleeve gastrectomy. Obes Surg 2016; 26: 1378-83.

13. Caballero Y, López-Tomassetti E, Castellot A, et al. Endoscopic management of a gastric leak after laparoscopic sleeve gastrectomy using the over-the-scope-clip $\left(\mathrm{Ovesco}^{\circledR}\right)$ system. Rev Esp Enferm Dig 2016; 108: 746-50.

14. Soufron J. Leak or fistula after sleeve gastrectomy: treatment with pigtail drain by the rendezvous technique. Obes Surg 2015; 25: 1979-80.

15. Leeds SG, Burdick JS. Management of gastric leaks after sleeve gastrectomy with endoluminal vacuum (E-Vac) therapy. Surg Obes Relat Dis 2016; 12: 1278-85.

Received: 17.11.2016, accepted: 24.05.2017. 